\title{
Diaphragm molding analysis of GFRTP using the finite element method
}

\author{
K. Tanaka ${ }^{1}$, M. Ogawa ${ }^{1}$, T. Katayama ${ }^{1} \&$ H. Sakamoto ${ }^{2}$ \\ ${ }^{1}$ Department of Biomedical Engineering, Doshisha University, Japan \\ ${ }^{2}$ Department of Mechanical Engineering, Kumamoto University, Japan
}

\begin{abstract}
Among fiber reinforced thermoplastics (FRTP) which have superior specific strength and stiffness, glass fiber reinforced thermoplastics (GFRTP) are generally used in the automotive industry due to its affordable prices. In diaphragm molding, which is a second process of GFRTP molding, FRTP are placed over the mold and are pressed by air pressure. This molding method can reduce the molding cost in comparison with the other press molding method because it can be molded by a single mold. However, its formability has not been clear yet. In this study, FEM analysis of diaphragm molding was performed and compared with the molded product. The position of wrinkles formed during the diaphragm molding can be predicted by FEM analysis. By shape comparison between the 3D data of the analyzed results and real products, the molded product was bigger than the analyzed result. This difference is considered to be the absence of the springback phenomenon of GFRTP during the cooling process in the FEM analysis.

Keywords: Glass Fiber Reinforced Thermoplastics (GFRTP), FEM, 3D scanner, wrinkles.
\end{abstract}

\section{Introduction}

Since the reduction of $\mathrm{CO}_{2}$ emissions from passenger cars is required in the automobile industries, the use of new materials that are lighter and stronger than conventional materials have been pushed forward in order to reduce the vehicle body weight [1-3]. Compared with the metal materials that are used for automobile body parts, fiber reinforced plastics (FRP) have high-specific strength and high-specific modulus. Among FRP, fiber reinforced thermoplastics (FRTP) whose matrix resin is thermoplastics provide recyclability and short cycle times 
compared to FRP whose matrix resin is thermoset resin [4]. In addition, FRTP have the ability to perform a secondary process because of their thermoplasticity. There are a variety of methods in the secondary process of FRTP: for example, matcheddie press molding, rubber molding and diaphragm molding [5]. Matched-die molding is a method to mold FRTP with a pair mold. While this method can apply high pressure to the materials, it requires mold cost of the top and bottom mold. In diaphragm molding (as shown in Fig. 1), FRTP are placed over the mold, and are pressed over by the diaphragm material using air pressure. Compared to conventional forming techniques such as matched-die molding, the diaphragm molding process is characterized by lower investment costs in terms of mold. However, since it is difficult for diaphragm molding to apply a blank holder force to FRTP, the diaphragm molded products are likely to have wrinkles. In diaphragm molding, the formability evaluation has been performed by the thickness comparison with the matched-die molding product and the in-plane wrinkling was reported to be attributed to the relative movements of the fibers in the two adjoining layers of a deforming laminate [6]. Although, formability in matcheddie molding has been clarified by FEM analysis [7, 8], the three-dimensional formability evaluation of diaphragm molding has not been performed. In this study, FEM analysis of diaphragm molding of GFRTP for hemisphere shape was performed to predict the wrinkle formation and the analyzed results were compared with the shape and 3D dimension of the molded products.

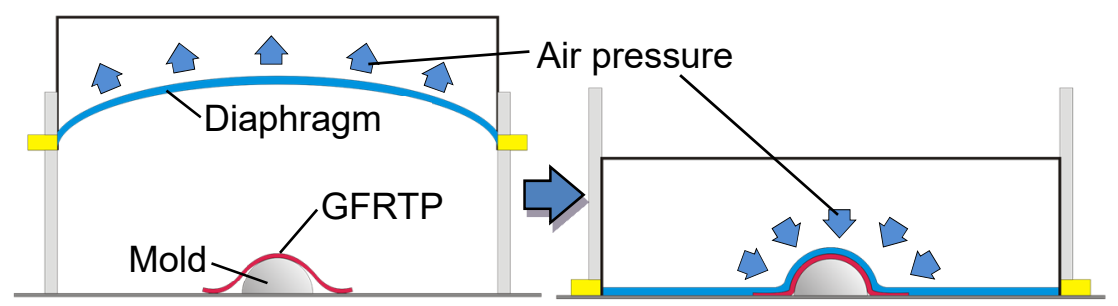

Figure 1: $\quad$ Schematic drawing of diaphragm molding.

\section{Material and experimental procedure}

\subsection{Material}

GFRTP (TEPEX dynalite 104-RGUD600 (1), Bond laminate Co.) consisting of glass fibers and PP were used as test materials. The glass fibers were woven with plain weave. Figure 2 shows the specimen of GFRTP. The specimen was a square, having a length of $150 \mathrm{~mm}$ on a side, a thickness of $0.5 \mathrm{~mm}$, and the fiber direction was $0^{\circ} / 90^{\circ}$. A silicon sheet for protecting the GFRTP surface after heating was a square having a length of $200 \mathrm{~mm}$ on a side and a thickness of $1 \mathrm{~mm}$. The mold has a hemisphere shape made of aluminum with a diameter of $100 \mathrm{~mm}$ (as shown in Fig. 3). 


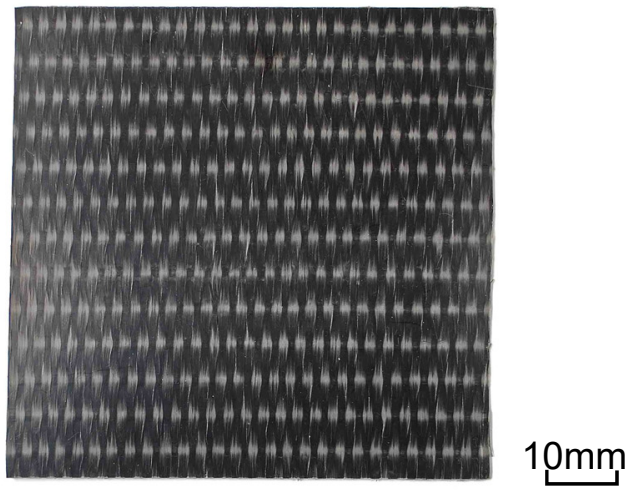

Figure 2: Specimen of GFRTP.

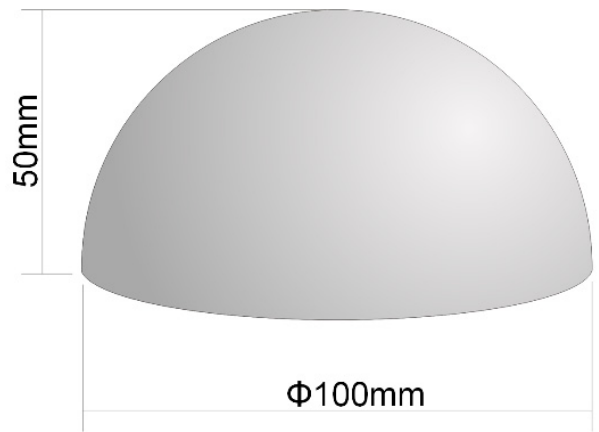

Figure 3: $\quad$ Mold.

\subsection{Molding method}

The diaphragm molding machine (MEMBRA5, LEIBROCK Co., Fig. 4), and the thermo heater (TH-5, LEIBROCK Co., Fig. 5) were used for molding. Molding

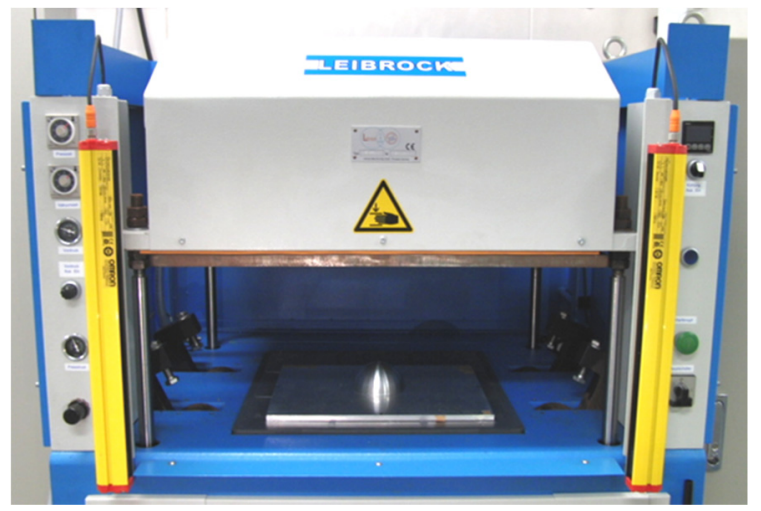

Figure 4: Diaphragm molding machine. 


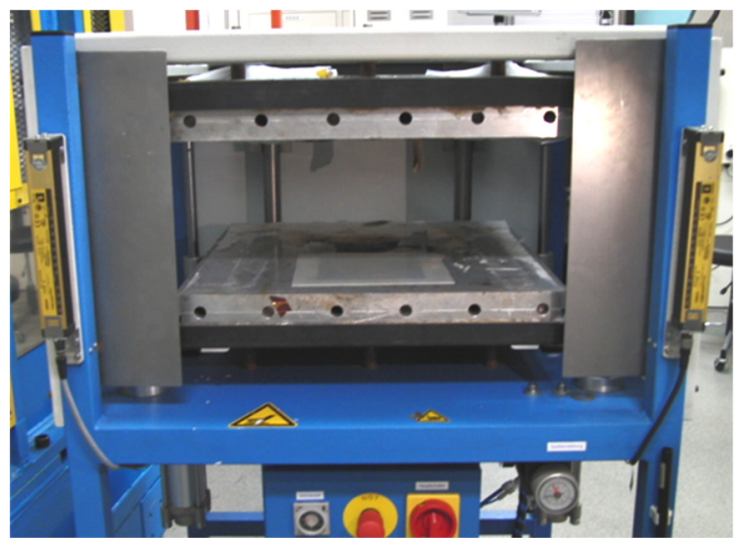

Figure 5: Thermo heater.

conditions were set as follows: $240^{\circ} \mathrm{C}$ in molding temperature, $0.3 \mathrm{MPa}$ in mold pressure, and $20 \mathrm{~s}$ in molding time. As the process of diaphragm molding, GFRTP were sandwiched between silicone sheets, and then heated to $240^{\circ} \mathrm{C}$ in the heater. When GFRTP reached the target temperature, GFRTP and silicone sheets were put on the mold and air pressure was applied from the top of the diaphragm material and the test specimen was molded.

\subsection{FEM analysis}

The composite material simulation software (PAM-FORM 2015.0, ESI Co.) was used for FEM analysis. Table 1 shows material properties which used for FEM analysis. The in-plane shear modulus obtained by the tensile test of $\pm 45^{\circ}$ GFRTP specimens under high temperature environment $\left(220^{\circ} \mathrm{C}\right)$. The stress-strain curve shown in Fig. 6, which were obtained by the tensile test of diaphragm and silicone sheet, were used for FEM analysis. Figure 7 shows the FEM model of the diaphragm forming. Diaphragm of rubber sheet was modeled as the square sheet having a length of one side of $500 \mathrm{~mm}$. The mold was set as a rigid body. Air pressure was applied from the top of the diaphragm material.

Table 1: $\quad$ Material property of the FEM model.

\begin{tabular}{|c|c|c|c|}
\hline Object & GFRTP & Diaphragm & Silicone sheet \\
\hline Size L*W*T $(\mathrm{mm})$ & $150 * 150 * 0.5$ & $500 * 500 * 5$ & $200 * 200 * 1$ \\
\hline Elements & 900 & 10000 & 1600 \\
\hline Node & 961 & 10201 & 1681 \\
\hline Fiber direction & $0 \% / 90^{\circ}$ & & \\
\hline Young's modulus (GPa) & \begin{tabular}{|l|l|}
$22\left(0^{\circ}\right)$ & $5\left(90^{\circ}\right)$ \\
\end{tabular} & & \\
\hline Poisson's ratio & & 0.499 & 0.499 \\
\hline In-plane shear modulus (MPa) & 1.2 & & \\
\hline Density $\rho\left(\mathrm{kg} / \mathrm{mm}^{\wedge} 3\right)$ & $1.8 \mathrm{E}-06$ & $1.15 \mathrm{E}-06$ & 1.23E-06 \\
\hline
\end{tabular}




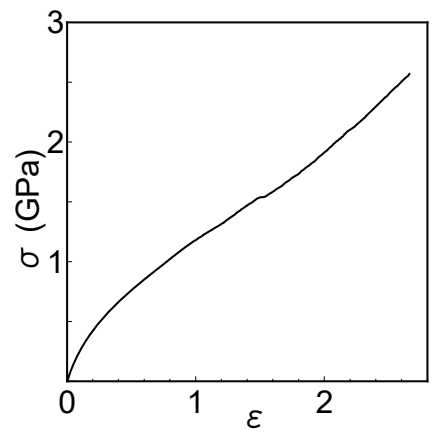

(a) Diaphragm.

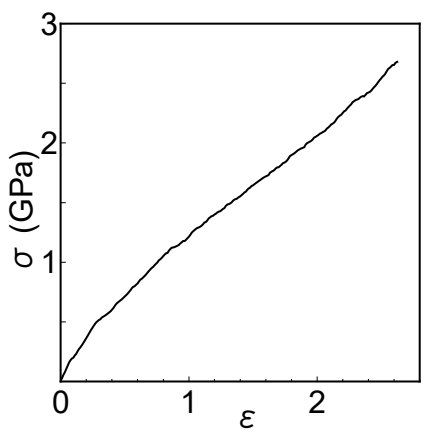

(b) Silicone sheet.

Figure 6: Stress-strain curve.

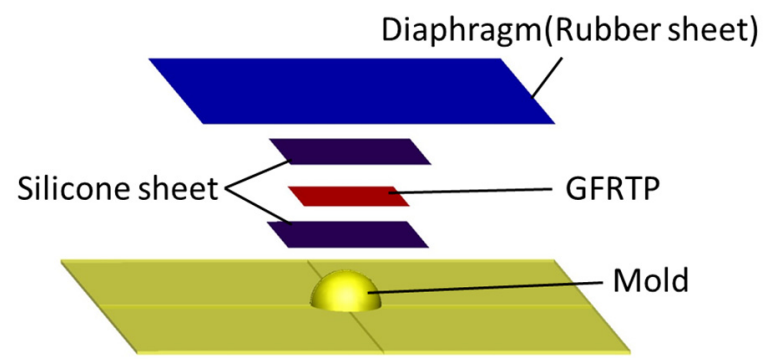

(a) Analyzed model.

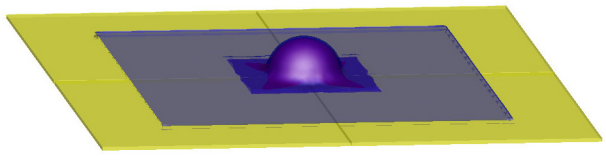

(b) Forming stage.

Figure 7: FEM model of diaphragm forming.

\subsection{Procedure of three-dimensional measurement for molded products}

For comparing the molded product and the analyzed result, the 3D scanner (Artec Eva, APT Co., Fig. 8) and the 3D data editing software (Artec Studio 9, APT Co.) were used. The molded product was scanned by the 3D scanner. An example of the scan data and analyzed result superimposed is shown in Fig. 9. Both vertexes are adjusted to the same position in the software, and cross sections were created at $5 \mathrm{~mm}$ intervals from the vertex. From these cross section data, the gap distance, that is the radius difference between the external form line of the scan data and the analyzed result, was measured and defined as the indicator of the formability. 


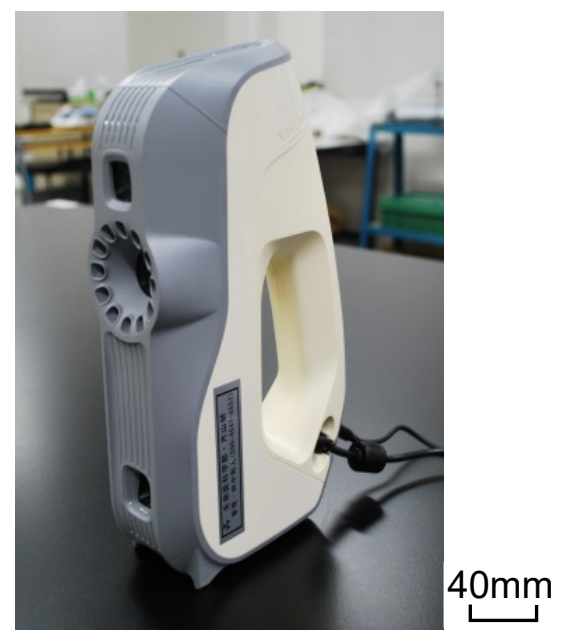

Figure 8: 3D scanner (Artec Eva).

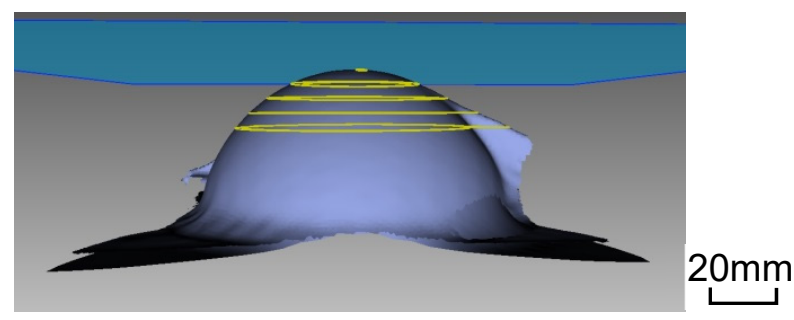

Figure 9: Superimposed scan data and analyzed result.

\section{Results and discussion}

The molded product created by the diaphragm molding and the analyzed result are shown in Fig. 10. In the molded product and the analyzed result, wrinkles were observed in the marked point in Fig. 10. The position of wrinkles formed can be predicted by the FEM analysis. However, the sizes of the wrinkles were different between the molded product and the analyzed result. For solving the issue, the mechanical properties of the compression direction that would cause wrinkling is considered to be included in the FEM analysis.

Figure 11 shows a slant view of the molded product scanned by the 3D scanner and the analyzed result. Figure 12 shows the superimposed image of the molded product and the analyzed result. The scan data of the molded product is shown with blue, and the analyzed result is shown with pink. Cross-sections of $10 \mathrm{~mm}$ from the vertex in the 3D data are shown in Fig. 13. The molded product is found to be larger than the analyzed result. Considering from the fact that the molded product is larger than the analyzed result, the spring-back phenomenon of the materials during the cooling time can be considered to influence their shape. 


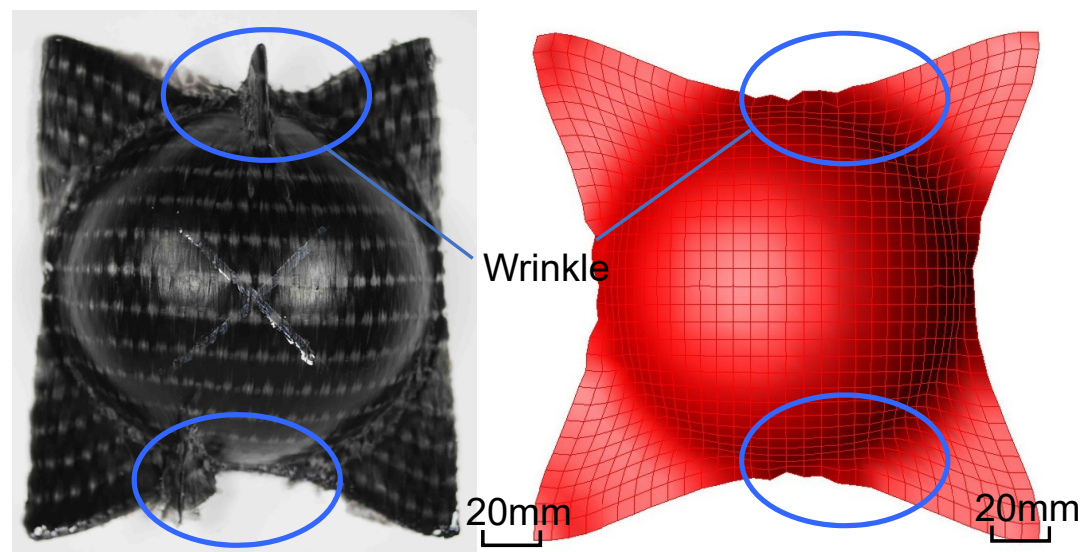

(a) Molded product.

(b) Analyzed result.

Figure 10: $\quad$ Plane view.

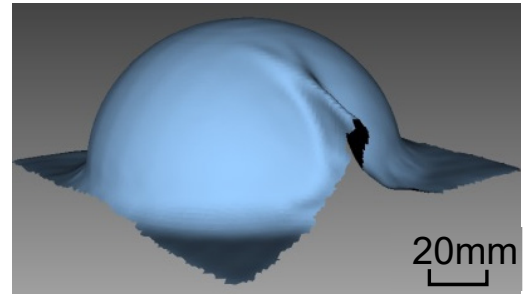

(a) Molded product (Scan data).

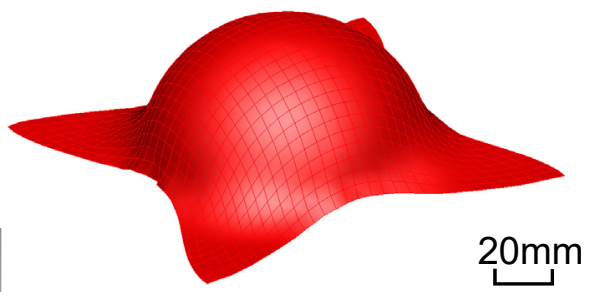

(b) Analyzed result.

Figure 11: Slant view.

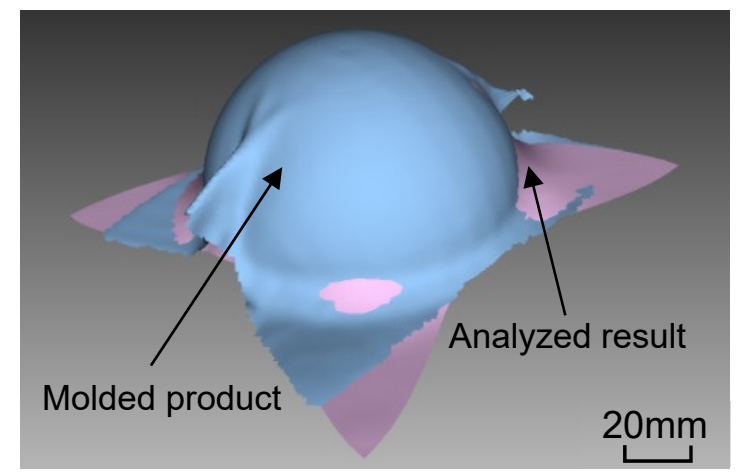

Figure 12: Comparison of molded product and analyzed result in 3D data. 


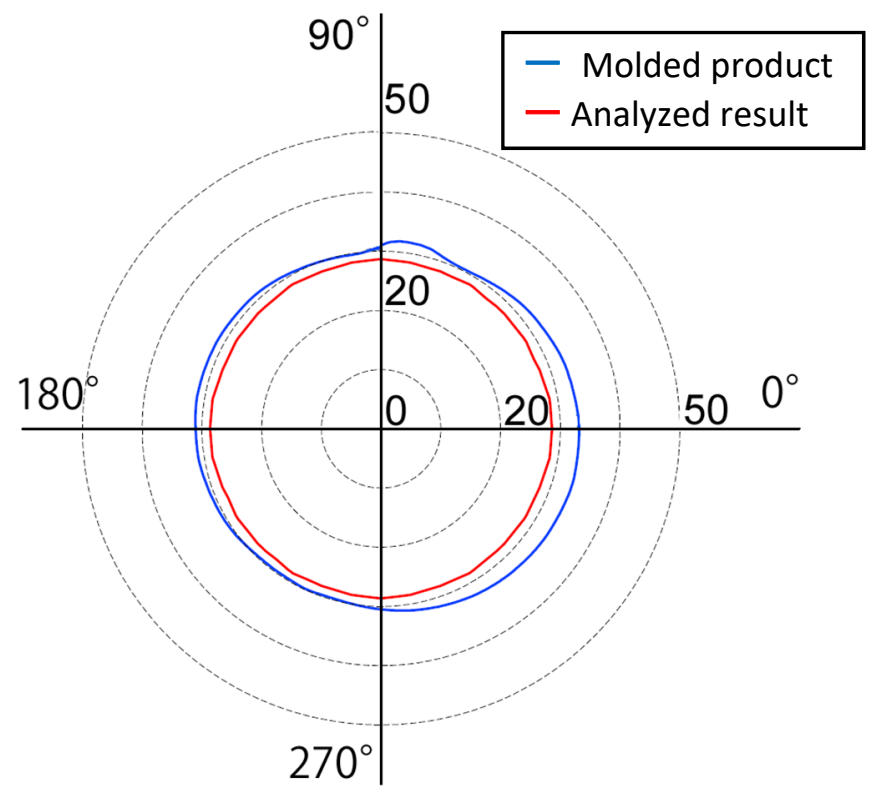

Figure 13: Sectional view of the molded product and the analyzed result $(10 \mathrm{~mm}$ from the vertex).

\section{Conclusions}

Diaphragm molding of GFRTP analysis was performed by FEM in order to reveal the formability. The analyzed results and the molded product with the diaphragm molding was compared based on 3D data. The investigation yielded the following conclusions:

1. The position of wrinkles formed during the diaphragm molding can be predicted by FEM analysis.

2. By the shape comparison between the $3 \mathrm{D}$ data of the analyzed results and real products, the molded product was bigger than the analyzed result.

\section{References}

[1] Yamane, M., Technical Collection of Carbon Fiber Reinforced Thermoplastics, Science \& Technology, pp. 16-28, 2015.

[2] Composites penetration growth in Automotive: towards mass production 2010-2020 trends and forecasts, JEC composites, pp. 13-22, 2011.

[3] Jacob, A., Car makers increase their use of composites, Reinforced Plastics, Vol. 48, No. 2, pp. 26-32, 2004.

[4] Uzawa, K., Takahashi, J., Kageyama, K., Uno, H., Okazumi, T. \& Ohsawa, I., Improvement in the Impact Energy Absorption of Recycled CFRP, ICCM17 Symposium, 2009. 
[5] Hopmann, C., Michaeli, W.\& Schoeldgen, R., Advanced Diaphragm Forming Process for High Temperature Thermoplastic Composite Applications, SAMPE Journal, Vol. 48, No. 2, pp. 24-29, 2012.

[6] Krebs, J., Friedrich, K. \& Bhattacharyya, D., A direct comparison of matcheddie versus diaphragm forming, Composite Part A, Vol. 129A, pp. 183-188, 1998.

[7] Nishi, M., Hirashima, T. \& Kurashiki, T., Dry Fabric Forming Analysis Considering the Influence of Tensions on In-plane Shear Behavior, Journal of the Society of Materials Science, Vol. 63, No. 5, pp. 380-385, 2014.

[8] Kusumi, K., Nomura, N. \& Maki, J., Formability and FEM Simulation of Steel Sheets in Hot Stamping Process, Nippon Steel Technical Report, No. 393, UDC 621.984, 2012. 